For the production of slaughter pigs by means of the hybridisation programme the economic evaluation of the own breeding work is very valuable. The detailed economic examinations have shown very clearly a tremendous space for the improvement process and an enormous return of investments put in this sphere. This follows from the number of final hybrids and from have shown very clearly a tremendous space for the improvement process and an enormous the amount of production of slaughter pigs per sow in elite herd. The profit function constructed for Czechoslovak conditions is presented and its use is shown. The solid knowledge of economic relations and functions in the whole process of production of slaughter pigs is thus becoming an unavoidable assumption for the management of the whole pig breeding.

\title{
Prüfungsmethoden, Populationsstruktur und ökonomische Parameter in ihrem Einfluss auf die Zuchtplanung beim Schwein
}

\section{E. NiEBEL. - Institut für Tierhaltung und Tierzüchtung der Universität Holenhein, BRD.}

Auf der Basis eines umfassenden EDV-Programms für die Reinzucht beim Schwein wird versucht, den wirtschaftlichen Erfolg der Planungsarbeit mit Hilfe der Kriterien des Zucht fortschritts je Jahr, des Kapitalwerts, der Pay-Off-Periode, der internen Verzinsung und der marginalen internen Verzinsung aufzuzeigen. Zur Quantifizierung des Zuchtziels wurde ein Gesamtzuchtwert aufgestellt, in dem die Leistungsmerkmale tägliche Zunahmen, Anteil wertvoller 'Teilstücke und Anzahl aufgezogener Ferkel berücksichtigt wurden. Die dafür notwendigen Wirtschaftlichkeitskoeffizienten wurden der Arbeit von BockENHOFF und al. (1976) entnommen. In den Planungsrechnungen wurde außerdem das genetische Niveau des Merkmals der Fleischhelligkeit konstant gehalten. Die Modellrechnungen erstreckten sich schwerpunktmäßig auf die Ermittlung der Effektivität unterschiedlicher Prüfungsmethoden von Ebern und Sauen sowie auf die Faktoren der Populationsstruktur.

Als vorläufige Ergebnisse der Modellrechnungen sind herauszustellen:

- Zuchtfortschritt, Kapitalwert, interne Verzinsung und Pay-Off-Periode führen bei der Beurteilung der Zweckmäßigkeit von Planungsalternativen beim Schwein zu ähnlicher Rangfolge

- die Voll- und Halbgeschwisterprüfung der Eber in Station in Kombination mit einer Eigenleistungsprüfung im Feld ergibt auch bei konstantem genetischen Niveau der Fleischhelligkeit einen befriedigenden Selektionserfolg, dagegen fällt die Eigenleistungsprüfung unter Restriktion in ihrer züchterischen Bedeutung stark ab

- durch die Einbeziehung der aus der Stationsprüfung verfügbaren Informationsquellen in die Zuchtwertschätzung der Sauen kann der Züchtungserfolg wesentlich gesteigert werden

- in der Zuchtplanung für die Reinzucht beim Schwein kommt den Faktoren der Populationsstruktur eine überragende Bedeutung zu. Auf die züchterische Relevanz der hier untersuchten Faktoren Prüfungskapazität in Station, Eber-Sauen-Verhältnis, Anzahl Selektionspfade bei der Genübertragung, Größe der Prüfgruppen und Zahl der Prüfgruppen je Eber wird hingewiesen.

\section{Zur EfFizienz der Zuchtwertschatzung von Jungsauen im Feld}

\section{W. Peschke und G. Averdunk. - Bayerische Landesanstalt für Tierzucht, 8oit Grub/BRD.}

In der bayerischen Herdbuchzucht (DL) wurden insgesamt 9216 Jungsauen im Feld geprüft und für die Merkmale der Eigenleistung (Zunahme, Speckdicke und Schinken) ein innerbetrieblicher Zuchtwert berechnet. Von 509 dieser Jungsauen wurden Nachkommen auf der Station geprüft. Die Parameter der Feldprüfung stimmen mit denen der Literatur weitgehend überein. Bei der Beurteilung der Selektionsdifferenzen ergeben sich Unterschiede zwischen Vollgeschwistern und Nachkommen. Als mögliche Ursache können in Frage kommen : die disassortative Anpaarung der selektierten Jungsauen, die unbefriedigende Erfassung der Zunahme bei hohen Lebendgewicht, sowie genetische Unterschiede zwischen Herden. Die Berücksichtigung der Geschisterinformation oder des genetischen Niveaus auf Grund der Stations-ergebnisse könnten diesen Index verbessern, doch sind hierfür weitere Untersuchungen notwendig. 